\title{
Abbreviations of Archival Sources
}

The following abbreviations are used in the endnotes, figures, maps, and tables. All citations are from the Colonial sections of each archive unless otherwise stated. I use the following system of archival citation: AGI-Escribania 3 I6b, 75: f. 2v means the cited information is in the Archivo General de Indias (Seville), the Escribania de Camara section, legajo number 3 I6b (all such numbers and letters being the archive's designations, not mine), expediente or pieza number 75: the verso side of folio 2. All archives were visited by me and documents accessed on site, unless otherwise stated.

AGAY Archivo General y Notaria Eclesiastica de la Arquidiócesis de Yucatán, Merida (accessed both on site and via LDS Church microfilm)

AGEC Archivo General del Estado de Campeche, Campeche

AGEY Archivo General del Estado de Yucatán, Merida

AGI Archivo General de Indias, Seville

AGMM Archivo General Militar de Madrid, Madrid

AGN Archivo General de la Nación, Mexico City

AGS Archivo General de Simancas, Simancas (accessed online and via Paso y Troncoso 1940)

AHAY Archivo Histórico de la Arquidiócesis de Yucatán, Merida

AHDC Archivo Histórico de la Diócesis de Campeche, Campeche

AHN Archivo Histórico Nacional, Madrid

AME Archivo de la Mitra Emeritense, Merida (accessed via Arrigunaga y Peón 1975, and Dumond and Dumond 1982)

ANEY Archivo Notarial del Estado de Yucatán, Merida (accessed both on site and via UTA microfilm, copies of which were acquired in 2006 by the Pennsylvania State University)

BL British Library (Rare Manuscript Room), London

BN Biblioteca Nacional, Mexico City 
CAIHY Centro de Apoyo a la Investigación Historica de Yucatán, Merida

CCA Colección Carrillo y Ancona, in CAIHY

CDH Colección de Documentos para la Historia de la Formación Social de Hispanoamérica, I493-1810 (see Konetzke 1953)

CI Cartas de Indias (Madrid, 1877 )

DCP Discurso Sobre la Constitución de las Provincias de Yucatán y Campeche (1766) in BN (Archivo Franciscano 55/I I 50) and in DHY

DHY Documentos para la Historia de Yucatán (Merida, 1936-38)

JCBL John Carter Brown Library, Providence

LLIU Lilly Library, Indiana University (accessed on site by Jason Frederick)

PRO -CO (Colonial Office), -A (Admiralty), (formerly) Public Record Office, (now called) National Archives, Kew (UK) (see Burdon I93I)

RAH Real Academia de la Historia, Madrid

TULAL Tulane University (New Orleans), Latin American Library Manuscript Collection

UTA University of Texas, Arlington (references to roll numbers are to microfilms of the ANEY) 
The Black Middle 
